\title{
Analytic description of the essential spectrum of a family of $3 \times 3$ operator matrices
}

\author{
T. H. Rasulov, N. A. Tosheva \\ Faculty of Physics and Mathematics, Bukhara State University M. Ikbol str. 11, 200100 Bukhara, Uzbekistan \\ rth@mail.ru, nargiza_n@mail.ru
}

DOI 10.17586/2220-8054-2019-10-5-511-519

\begin{abstract}
We consider the family of $3 \times 3$ operator matrices $H(K), K \in \mathbb{T}^{\mathrm{d}}:=(-\pi ; \pi]^{\mathrm{d}}$ arising in the spectral analysis of the energy operator of the spin-boson model of radioactive decay with two bosons on the torus $\mathbb{T}^{\mathrm{d}}$. We obtain an analog of the Faddeev equation for the eigenfunctions of $H(K)$. An analytic description of the essential spectrum of $H(K)$ is established. Further, it is shown that the essential spectrum of $H(K)$ consists the union of at most three bounded closed intervals.
\end{abstract}

Keywords: family of operator matrices, generalized Friedrichs model, bosonic Fock space, annihilation and creation operators, channel operator, decomposable operator, fiber operators, the Faddeev equation, essential spectrum, Weyl criterion.

Received: 6 October 2019

\section{Introduction}

In statistical physics [1], solid-state physics [2] and the theory of quantum fields [3], one considers systems, where the number of quasi-particles is not fixed. Their number can be unbounded, as in the case of full spin-boson models (infinite operator matrix) [4] or bounded, as in the case of "truncated" spin-boson models (finite operator matrix) [1,5-8]. Often, the number of particles can be arbitrarily large as in cases involving photons, in other cases, such as scattering of spin waves on defects, scattering massive particles and chemical reactions, there are only participants at any given time, though their number can be changed.

Recall that the study of systems describing $N$ particles in interaction, without conservation of the number of particles is reduced to the investigation of the spectral properties of self-adjoint operators, acting in the cut subspace $\mathcal{H}^{(N)}$ of Fock space, consisting of $n \leq N$ particles $[1-3,9]$.

The essential spectrum of the Hamiltonians (matrix operators) in the Fock space are the most actively studied objects in operator theory. One of the important problems in the spectral analysis of these operators is to describe the location of the essential spectrum. One of the well-known methods for the investigating the location of essential spectra of operator matrices are Weyl criterion and the Hunziker-van Winter-Zhislin (HWZ) theorem. Using these methods, in many works, the essential spectrum of the $3 \times 3$ and $4 \times 4$ lattice operator matrices are studied, see e.g., [10-13]. In particular, in [13] it is described the essential spectrum of $4 \times 4$ operator matrix by the spectrum of the corresponding channel operators and proved the HWZ theorem. In [9], geometric and commutator techniques have been developed in order to find the location of the spectrum and to prove absence of singular continuous spectrum for Hamiltonians without conservation of the particle number.

In the present paper, we consider the family of $3 \times 3$ operator matrices $H(K), K \in \mathbb{T}^{\mathrm{d}}$ associated with the lattice systems describing two identical bosons and one particle, another nature in interactions, without conservation of the number of particles. This operator acts in the direct sum of zero-, one- and two-particle subspaces of the bosonic Fock space and it is a lattice analog of the spin-boson Hamiltonian [1]. In order to study the location of the essential spectrum of $H(K)$, we first introduce the notion of channel operator $H_{\mathrm{ch}}(K)$ corresponding to $H(K)$. Using the theorem on the spectrum of decomposable operators we describe the spectrum of $H_{\mathrm{ch}}(K)$ via the spectrum of a family of generalized Friedrichs models $h(K, k), K, k \in \mathbb{T}^{\mathrm{d}}$. Then, we prove that the essential spectrum of $H(K)$ is coincide with the spectrum of $H_{\mathrm{ch}}(K)$ and show that the set $\sigma_{\mathrm{ess}}(H(K))$ consists the union of at most 3 bounded closed intervals. Further, we define the new so-called two- and three-particle branches of $\sigma_{\mathrm{ess}}(H(K))$.

We point out that the operator $H(K)$ has been considered before in $[14,15]$ for a fixed $K \in \mathbb{T}^{\mathrm{d}}$ and studied its essential and discrete spectrum. In this paper, we investigate the essential spectrum of $H(K)$ depending on $K \in \mathbb{T}^{\mathrm{d}}$. It is remarkable that, the essential spectrum and the number of the eigenvalues of a slightly simpler version of $H(K)$ were studied in [16] and the result for the location of the essential spectrum were announced without proof.

The present paper is organized as follows. Section 1 is an introduction to the whole work. In Section 2, the operator matrices $H(K), K \in \mathbb{T}^{\mathrm{d}}$ are described as the family of bounded self-adjoint operators in the direct sum of zero-, one- and two-particle subspaces of the bosonic Fock space and the main aims of the paper are stated. The family of generalized Friedrichs model is introduced and its spectrum is established in Section 3. In Section 4, we determine the channel operator $H_{\mathrm{ch}}(K)$ corresponding to $H(K)$ and define its spectrum. The next Section is devoted to the 
derivation of the Faddeev equation for the eigenvectors of $H(K)$. In Section 6, we investigate the essential spectrum of $H(K)$ and its new branches.

Throughout this paper, we use the following notation. If $A$ is a linear bounded self-adjoint operator from one Hilbert space to another, then $\sigma(A)$ denotes its spectrum, $\sigma_{\text {ess }}(A)$ its essential spectrum and $\sigma_{\text {disc }}(A)$ its discrete spectrum.

\section{Family of $3 \times 3$ operator matrices and its relation with spin-boson models}

Let us introduce some notations used in this work. Let $\mathbb{T}^{\mathrm{d}}$ be the d-dimensional torus, the cube $(-\pi, \pi]^{\mathrm{d}}$ with appropriately identified sides equipped with its Haar measure. Let $\mathcal{H}_{0}:=\mathbb{C}$ be the field of complex numbers (channel $1), \mathcal{H}_{1}:=L^{2}\left(\mathbb{T}^{\mathrm{d}}\right)$ be the Hilbert space of square integrable (complex) functions defined on $\mathbb{T}^{\mathrm{d}}$ (channel 2) and $\mathcal{H}_{2}:=L_{\text {sym }}^{2}\left(\left(\mathbb{T}^{\mathrm{d}}\right)^{2}\right)$ stands for the subspace of $L^{2}\left(\left(\mathbb{T}^{\mathrm{d}}\right)^{2}\right)$ consisting of symmetric functions (with respect to the two variables) (channel 3). We denote by $\mathcal{H}$ the direct sum of spaces $\mathcal{H}_{0}, \mathcal{H}_{1}$ and $\mathcal{H}_{2}$, that is, $\mathcal{H}=\mathcal{H}_{0} \oplus \mathcal{H}_{1} \oplus \mathcal{H}_{2}$. The spaces $\mathcal{H}_{0}, \mathcal{H}_{1}$ and $\mathcal{H}_{2}$ are called zero-, one- and two-particle subspaces of a bosonic Fock space $\mathcal{F}_{\mathrm{s}}\left(L^{2}\left(\mathbb{T}^{\mathrm{d}}\right)\right)$ over $L^{2}\left(\mathbb{T}^{\mathrm{d}}\right)$, respectively, and the Hilbert space $\mathcal{H}$ is called the truncated Fock space or the three-particle cut subspace of the Fock space. We write elements $f$ of the space $\mathcal{H}$ in the form, $f=\left(f_{0}, f_{1}, f_{2}\right), f_{i} \in \mathcal{H}_{i}, i=0,1,2$ and for any two elements $f=\left(f_{0}, f_{1}, f_{2}\right), g=\left(g_{0}, g_{1}, g_{2}\right) \in \mathcal{H}$ their scalar product is defined by:

$$
(f, g):=f_{0} \overline{g_{0}}+\int_{\mathbb{T}^{\mathrm{d}}} f_{1}(t) \overline{g_{1}(t)} d t+\int_{\left(\mathbb{T}^{\mathrm{d}}\right)^{2}} f_{2}(s, t) \overline{g_{2}(s, t)} d s d t .
$$

In the present paper we study the essential spectrum of the family of $3 \times 3$ tridiagonal operator matrices:

$$
H(K):=\left(\begin{array}{ccc}
H_{00}(K) & H_{01} & 0 \\
H_{01}^{*} & H_{11}(K) & H_{12} \\
0 & H_{12}^{*} & H_{22}(K)
\end{array}\right), \quad K \in \mathbb{T}^{\mathrm{d}}
$$

in the Hilbert space $\mathcal{H}$. The matrix entries $H_{i i}(K): \mathcal{H}_{i} \rightarrow \mathcal{H}_{i}, i=0,1,2$ and $H_{i j}: \mathcal{H}_{j} \rightarrow \mathcal{H}_{i}, i<j, i, j=0,1,2$ are given by:

$$
\begin{gathered}
H_{00}(K) f_{0}=w_{0}(K) f_{0}, \quad H_{01} f_{1}=\int_{\mathbb{T}^{\mathrm{d}}} v_{0}(t) f_{1}(t) d t \\
\left(H_{11}(K) f_{1}\right)(p)=w_{1}(K ; p) f_{1}(p), \quad\left(H_{12} f_{2}\right)(p)=\int_{\mathbb{T}^{\mathrm{d}}} v_{1}(t) f_{2}(p, t) d t, \\
\left(H_{22}(K) f_{2}\right)(p, q)=w_{2}(K ; p, q) f_{2}(p, q), \quad f_{i} \in \mathcal{H}_{i}, \quad i=0,1,2 .
\end{gathered}
$$

Throughout the paper, we assume that the parameter functions $w_{0}(\cdot), v_{i}(\cdot), i=0,1 ; w_{1}(\cdot ; \cdot)$ and $w_{2}(\cdot ; \cdot, \cdot)$ are real-valued continuous functions on $\mathbb{T}^{\mathrm{d}} ;\left(\mathbb{T}^{\mathrm{d}}\right)^{2}$ and $\left(\mathbb{T}^{\mathrm{d}}\right)^{3}$, respectively. In addition, for any fixed $K \in \mathbb{T}^{\mathrm{d}}$ the function $w_{2}(K ; \cdot, \cdot)$ is a symmetric; that is, the equality $w_{2}(K ; p, q)=w_{2}(K ; q, p)$ holds for any $p, q \in \mathbb{T}^{\mathrm{d}}$.

Under these assumptions the operator $H(K)$ is bounded and self-adjoint.

We remark that the operators $H_{01}$ and $H_{12}$ resp. $H_{01}^{*}$ and $H_{12}^{*}$ are called annihilation resp. creation operators, respectively. A trivial verification shows that:

$$
\begin{aligned}
& H_{01}^{*}: \mathcal{H}_{0} \rightarrow \mathcal{H}_{1}, \quad\left(H_{01}^{*} f_{0}\right)(p)=v_{0}(p) f_{0}, \quad f_{0} \in \mathcal{H}_{0} ; \\
& H_{12}^{*}: \mathcal{H}_{1} \rightarrow \mathcal{H}_{2}, \quad\left(H_{12}^{*} f_{1}\right)(p, q)=\frac{1}{2}\left(v_{1}(p) f_{1}(q)+v_{1}(q) f_{1}(p)\right), \quad f_{1} \in \mathcal{H}_{1} .
\end{aligned}
$$

These operators have widespread applications in quantum mechanics, notably in the study of quantum harmonic oscillators and many-particle systems [17]. An annihilation operator lowers the number of particles in a given state by one. A creation operator increases the number of particles in a given state by one, and it is the adjoint of the annihilation operator. In many subfields of physics and chemistry, the use of these operators instead of wavefunctions is known as second quantization. In this paper we consider the case, where the number of annihilations and creations of the particles of the considering system is equal to 1 . This means that $H_{i j} \equiv 0$ for all $|i-j|>1$.

The family of operator matrices of this form plays a key role for the study of the energy operator of the spinboson model with two bosons on the torus. In fact, the latter is a $6 \times 6$ operator matrix which is unitary equivalent to a $2 \times 2$ block diagonal operator with two copies of a particular case of $H(K)$ on the diagonal, see [6]. Consequently, the essential spectrum and finiteness of discrete eigenvalues of the spin-boson Hamiltonian are determined by the corresponding spectral information on the operator matrix $H(K)$ in (2.1).

Independently of whether the underlying domain is a torus or the whole space or the whole space $\mathbb{R}^{\mathrm{d}}$, the full spinboson Hamiltonian is an infinite operator matrix in Fock space for which rigorous results are very hard to obtain. One 
line of attack is to consider the compression to the truncated Fock space with a finite $N$ of bosons, and in fact, most of the existing literature concentrates on the case $N \leq 2$. For the case of $\mathbb{R}^{\mathrm{d}}$, there some exceptions, see e.g. [4,5] for arbitrary finite $N$ and [8] for $N=3$, where a rigorous scattering theory was developed for small coupling constants.

For the case when the underlying domain is a torus, the spectral properties $H(K)$ for a fixed $K$ were investigated in $[6,10,13-15]$, see also the references therein. The results obtained in this paper for all $K$ will play important role when we study the problem of finding subset $\Lambda \subset \mathbb{T}^{\mathrm{d}}$, such that the operator matrices $H(K)$ have a finite number of or infinitely many eigenvalues for all $K \in \Lambda$.

It is well-known that the three-particle discrete Schrödinger operator $\widehat{H}$ in the momentum representation is the bounded self-adjoint operator on the Hilbert space $L_{2}\left(\left(\mathbb{T}^{\mathrm{d}}\right)^{3}\right)$. Introducing the total quasimomentum $K \in \mathbb{T}^{\mathrm{d}}$ of the system, it is easy to see that the operator $\widehat{H}$ can undergo decomposition to give the direct integral of the family $\left\{\widehat{H}(K), K \in \mathbb{T}^{\mathrm{d}}\right\}$ of self-adjoint operators $[18,19]$ :

$$
\widehat{H}=\int_{\mathbb{T}^{\mathrm{d}}} \oplus \widehat{H}(K) d K,
$$

where the operator $\widehat{H}(K)$ acts on the Hilbert space $L_{2}\left(\Gamma_{K}\right)\left(\Gamma_{K} \subset\left(\mathbb{T}^{\mathrm{d}}\right)^{2}\right.$ is some manifold).

Observe that $H(K)$ enjoys the main spectral properties of the three-particle discrete Schrödinger operator $\widehat{H}(K)$ (see $[18,19])$, and the generalized Friedrichs model plays the role of the two-particle discrete Schrödinger operator. For this reason, the Hilbert space $\mathcal{H}$ is called the three-particle cut subspace of the Fock space, while the operator matrix $H(K)$ the Hamiltonian of the system with at most three particles on a lattice.

The main aims of the present paper are as follows:

(i) to investigate the spectrum of a family of generalized Friedrichs model;

(ii) to introduce the channel operator $H_{\mathrm{ch}}(K)$ corresponding to $H(K)$ and describe its spectrum;

(iii) to obtain an analog of the Faddeev equation for eigenvectors of $H(K)$;

(iv) to show that the essential spectrum of $H(K)$ is coincident with the spectrum of $H_{\mathrm{ch}}(K)$;

(v) to prove that the essential spectrum of $H(K)$ consists at most three bounded closed intervals;

(vi) to define the new branches of $\sigma_{\text {ess }}(H(K))$.

The next sections are devoted to the discussion of these problems.

\section{Family of generalized Friedrichs models and its spectrum}

To study the spectral properties of the operator $H(K)$, we introduce a family of bounded self-adjoint operators (generalized Friedrichs models) $h(K, k), K, k \in \mathbb{T}^{\mathrm{d}}$, which acts in the Hilbert space $\mathcal{H}_{0} \oplus \mathcal{H}_{1}$ as $2 \times 2$ operator matrices:

$$
h(K, k):=\left(\begin{array}{cc}
h_{00}(K, k) & h_{01} \\
h_{01}^{*} & h_{11}(K, k)
\end{array}\right),
$$

with matrix elements:

$$
\begin{aligned}
& h_{00}(K, k) f_{0}=w_{1}(K, k) f_{0}, h_{01} f_{1}=\frac{1}{\sqrt{2}} \int_{\mathbb{T}^{\mathrm{d}}} v_{1}(s) f_{1}(s) d s, \\
& \left(h_{11}(K, k) f_{1}\right)(q)=w_{2}(K ; k, q) f_{1}(q), \quad f_{i} \in \mathcal{H}_{i}, \quad i=0,1 .
\end{aligned}
$$

Here,

$$
h_{01}^{*}: \mathcal{H}_{0} \rightarrow \mathcal{H}_{1}, \quad\left(h_{01}^{*} f_{0}\right)(p)=v_{1}(p) f_{0}, \quad f_{0} \in \mathcal{H}_{0}
$$

Next we study some spectral properties of the family $h(K, k)$, given by (3.1), which plays a crucial role in the study of the essential spectrum of $H(K)$. We notice that the spectrum, usual eigenvalues, threshold eigenvalues and virtual levels of the typical models for a fixed $K, k \in \mathbb{T}^{\mathrm{d}}$ have been studied in many works, see for example [14,15,20].

Let the operator $h_{0}(K, k), K, k \in \mathbb{T}^{\mathrm{d}}$ acts in $\mathcal{H}_{0} \oplus \mathcal{H}_{1}$ as:

$$
h_{0}(K, k):=\left(\begin{array}{cc}
0 & 0 \\
0 & h_{11}(K, k)
\end{array}\right) .
$$

The perturbation $h(K, k)-h_{0}(K, k)$ of the operator $h_{0}(K, k)$ is a self-adjoint operator of rank 2 , and thus, according to the Weyl theorem, the essential spectrum of the operator $h(K, k)$ coincides with the essential spectrum of $h_{0}(K, k)$. It is evident that:

$$
\sigma_{\mathrm{ess}}\left(h_{0}(K, k)\right)=\left[E_{\min }(K, k) ; E_{\max }(K, k)\right],
$$


where the numbers $E_{\min }(K, k)$ and $E_{\max }(K, k)$ are defined by:

$$
E_{\min }(K, k):=\min _{q \in \mathbb{T}^{d}} w_{2}(K ; k, q) \quad \text { and } \quad E_{\max }(K, k):=\max _{q \in \mathbb{T}^{d}} w_{2}(K ; k, q) .
$$

This yields $\sigma_{\text {ess }}(h(K, k))=\left[E_{\min }(K, k) ; E_{\max }(K, k)\right]$.

Remark 3.1. We remark that for some $K, k \in \mathbb{T}^{\mathrm{d}}$ the essential spectrum of $h(K, k)$ may degenerate to the set consisting of the unique point $\left[E_{\min }(K, k) ; E_{\min }(K, k)\right]$ and hence we can not state that the essential spectrum of $h(K, k)$ is absolutely continuous for any $K, k \in \mathbb{T}^{\mathrm{d}}$. For example, this is the case if the function $w_{2}(\cdot ; \cdot, \cdot)$ is of the form:

$$
\begin{gathered}
w_{2}(K ; k, q)=\varepsilon(k)+\varepsilon(q)+\varepsilon(K-k-q), \\
K=\overline{0}:=(0, \ldots, 0), k=\bar{\pi}:=\begin{array}{l}
(\pi, \ldots, \pi) \in \mathbb{T}^{\mathrm{d}} \text { and } \\
\varepsilon(q)=\sum_{i=1}^{\mathrm{d}}\left(1-\cos q_{i}\right), \quad q=\left(q_{1}, \ldots, q_{\mathrm{d}}\right) \in \mathbb{T}^{\mathrm{d}} .
\end{array}
\end{gathered}
$$

Then $\sigma_{\text {ess }}(h(\overline{0}, \bar{\pi}))=\{4 \mathrm{~d}\}$.

For any fixed $K, k \in \mathbb{T}^{\mathrm{d}}$ we define an analytic function $\Delta_{K}(k ; \cdot)$ (the Fredholm determinant associated with the operator $h(K, k))$ in $\mathbb{C} \backslash\left[E_{\min }(K, k) ; E_{\max }(K, k)\right]$ by:

$$
\Delta_{K}(k ; z):=w_{1}(K ; k)-z-\frac{1}{2} \int_{\mathbb{T}^{\mathrm{d}}} \frac{v_{1}^{2}(t) d t}{w_{2}(K ; k, t)-z} .
$$

The following lemma [14] is a simple consequence of the Birman-Schwinger principle and the Fredholm theorem.

Lemma 3.2. For any $K, k \in \mathbb{T}^{\mathrm{d}}$ the operator $h(K, k)$ has an eigenvalue $z(K, k) \in \mathbb{C} \backslash\left[E_{\min }(K, k) ; E_{\max }(K, k)\right]$ if and only if $\Delta_{K}(k ; z(K, k))=0$.

From Lemma 3.2 it follows that for the discrete spectrum of $h(K, k)$ the equality:

$$
\sigma_{\text {disc }}(h(K, k))=\left\{z \in \mathbb{C} \backslash\left[E_{\min }(K, k) ; E_{\max }(K, k)\right]: \Delta_{K}(k ; z)=0\right\}
$$

holds.

The following lemma describes the number and location of the eigenvalues of $h(K, k)$.

Lemma 3.3. For any fixed $K, k \in \mathbb{T}^{d}$ the operator $h(K, k)$ has no more than one simple eigenvalue lying on the l.h.s. (resp. r.h.s.) of $E_{\min }(K, k)$ (resp. $E_{\max }(K, k)$ ).

The proof of Lemma 3.3 is an elementary and it follows from the fact that for any fixed $K, k \in \mathbb{T}^{\mathrm{d}}$ the function $\Delta_{K}(k ; \cdot)$ is a monotonically decreasing on $\left(-\infty ; E_{\min }(K, k)\right)$ and $\left(E_{\max }(K, k) ;+\infty\right)$.

\section{The spectrum of channel operator corresponding to $H(K)$}

In this section, we define the channel operator $H_{\mathrm{ch}}(K)$ corresponding to $H(K)$ and describe its spectrum by the spectrum of the family of operators $h(K, k), K, k \in \mathbb{T}^{\mathrm{d}}$, defined by (3.1).

We introduce so-called channel operator $H_{\mathrm{ch}}(K)$ acting in $L^{2}\left(\mathbb{T}^{\mathrm{d}}\right) \oplus L^{2}\left(\left(\mathbb{T}^{\mathrm{d}}\right)^{2}\right)$ as a family of $2 \times 2$ operator matrices:

$$
H_{\mathrm{ch}}(K):=\left(\begin{array}{cc}
H_{11}(K) & \frac{1}{\sqrt{2}} H_{12} \\
\frac{1}{\sqrt{2}} H_{12}^{*} & H_{22}(K)
\end{array}\right), \quad K \in \mathbb{T}^{\mathrm{d}} .
$$

It is important that for this case the operator $H_{12}^{*}$ is defined as follows:

$$
H_{12}^{*}: L^{2}\left(\mathbb{T}^{\mathrm{d}}\right) \rightarrow L^{2}\left(\left(\mathbb{T}^{\mathrm{d}}\right)^{2}\right), \quad\left(H_{12}^{*} f_{1}\right)(p, q)=v_{1}(q) f_{1}(p), \quad f_{1} \in L^{2}\left(\mathbb{T}^{\mathrm{d}}\right) .
$$

Under these assumptions the operator $H_{\mathrm{ch}}(K)$ is bounded and self-adjoint.

We set:

$$
\begin{aligned}
& m_{K}:=\min _{p, q \in \mathbb{T}^{\mathrm{d}}} w_{2}(K ; p, q), \quad M_{K}:=\max _{p, q \in \mathbb{T}^{\mathrm{d}}} w_{2}(K ; p, q), \\
& \Lambda_{K}:=\bigcup_{k \in \mathbb{T}^{\mathrm{d}}} \sigma_{\operatorname{disc}}(h(K, k)), \quad \Sigma_{K}:=\left[m_{K} ; M_{K}\right] \cup \Lambda_{K} .
\end{aligned}
$$

Here, by Lemma 3.2, we may define the set $\Lambda_{K}$ as the set of all complex numbers $z \in \mathbb{C} \backslash\left[E_{\min }(K, k) ; E_{\max }(K, k)\right]$ such that the equality $\Delta_{K}(k ; z)=0$ holds for some $k \in \mathbb{T}^{\mathrm{d}}$.

The spectrum of the operator $H_{\mathrm{ch}}(K)$ can be precisely described by the spectrum of the family $h(K, k)$ of generalized Friedrichs models as well as in the following assertion. 
Theorem 4.1. The operator $H_{\mathrm{ch}}(K)$ has a purely essential spectrum and for its spectrum the equality $\sigma\left(H_{\mathrm{ch}}(K)\right)=$ $\Sigma_{K}$ holds.

Proof. It is clear that the operator $H_{\mathrm{ch}}(K)$ commutes with any multiplication operator $U_{\alpha}$ by the bounded function $\alpha(\cdot)$ on $\mathbb{T}^{\mathrm{d}}$ :

$$
U_{\alpha}\left(\begin{array}{c}
g_{1}(p) \\
g_{2}(p, q)
\end{array}\right)=\left(\begin{array}{c}
\alpha(p) g_{1}(p) \\
\alpha(p) g_{2}(p, q)
\end{array}\right),\left(\begin{array}{c}
g_{1} \\
g_{2}
\end{array}\right) \in L^{2}\left(\mathbb{T}^{\mathrm{d}}\right) \oplus L^{2}\left(\left(\mathbb{T}^{\mathrm{d}}\right)^{2}\right) .
$$

Therefore the decomposition of the space $L^{2}\left(\mathbb{T}^{\mathrm{d}}\right) \oplus L^{2}\left(\left(\mathbb{T}^{\mathrm{d}}\right)^{2}\right)$ into the direct integral:

$$
L^{2}\left(\mathbb{T}^{\mathrm{d}}\right) \oplus L^{2}\left(\left(\mathbb{T}^{\mathrm{d}}\right)^{2}\right)=\int_{\mathbb{T}^{\mathrm{d}}} \oplus\left(\mathcal{H}_{0} \oplus \mathcal{H}_{1}\right) d k
$$

yields the decomposition into the direct integral:

$$
H_{\mathrm{ch}}(K)=\int_{\mathbb{T}^{\mathrm{d}}} \oplus h(K, k) d k
$$

where the fiber operators (a family of the generalized Friedrichs models) $h(K, k)$ are defined by (3.1). We note that identical fibers appear in the direct integral in decomposition (4.2). Then the theorem on the spectrum of decomposable operators [21] gives the equality:

$$
\sigma\left(H_{\mathrm{ch}}(K)\right)=\bigcup_{k \in \mathbb{T}^{\mathrm{d}}} \sigma(h(K, k))
$$

The definition of the set $\Lambda_{K}$ and the equality:

$$
\bigcup_{k \in \mathbb{T}^{\mathrm{d}}}\left[E_{\min }(K, k) ; E_{\max }(K, k)\right]=\left[m_{K} ; M_{K}\right]
$$

imply the equality:

$$
\bigcup_{k \in \mathbb{T}^{\mathrm{d}}} \sigma(h(K, k))=\Sigma_{K}
$$

Now, the equalities (4.4) and (4.5) complete the proof.

\section{The Faddeev equation and main property}

In this section, we derive an analog of the Faddeev type system of integral equations for the eigenvectors corresponding to the discrete eigenvalues (isolated eigenvalues with finite multiplicity) of $H(K)$, which plays a crucial role in our analysis of the spectrum of $H(K)$.

For any fixed $K \in \mathbb{T}^{\mathrm{d}}$ and $z \in \mathbb{C} \backslash \Sigma_{K}$ we introduce a $2 \times 2$ block operator matrix $T(K, z)$ acting in $\mathcal{H}_{0} \oplus \mathcal{H}_{1}$ as:

$$
T(K, z):=\left(\begin{array}{cc}
T_{00}(K, z) & T_{01}(K, z) \\
T_{10}(K, z) & T_{11}(K, z)
\end{array}\right)
$$

where the entries $T_{i j}(K, z): \mathcal{H}_{j} \rightarrow \mathcal{H}_{i}, i, j=0,1$ are defined by:

$$
\begin{gathered}
T_{00}(K, z) g_{0}=\left(1+w_{0}(K)-z\right) g_{0}, \quad T_{01}(K, z) g_{1}=\int_{\mathbb{T}^{d}} v_{0}(t) g_{1}(t) d t ; \\
\left(T_{10}(K, z) g_{0}\right)(p)=-\frac{v_{0}(p) g_{0}}{\Delta_{K}(p ; z)}, \quad\left(T_{11}(K, z) g_{1}\right)(p)=\frac{v_{1}(p)}{2 \Delta_{K}(p ; z)} \int_{\mathbb{T}^{d}} \frac{v_{1}(t) g_{1}(t) d t}{w_{2}(K ; p, t)-z} .
\end{gathered}
$$

Here, $g_{i} \in \mathcal{H}_{i}, i=0,1$. We note that for each $K \in \mathbb{T}^{\mathrm{d}}$ and $z \in \mathbb{C} \backslash \Sigma_{K}$ the entries $T_{00}(K, z), T_{01}(K, z)$ and $T_{10}(K, z)$ are rank 1 operators, the integral operator $T_{11}(K, z)$ belongs to the Hilbert-Schmidt class and therefore, $T(K, z)$ is a compact operator.

The following theorem is an analog of the well-known Faddeev's result for the operator $H(K)$ and establishes a connection between eigenvalues of $H(K)$ and $T(K, z)$.

Theorem 5.1. The number $z \in \mathbb{C} \backslash \Sigma_{K}$ is an eigenvalue of the operator $H(K)$ if and only if the number $\lambda=1$ is an eigenvalue of the operator $T(K, z)$. Moreover the eigenvalues $z$ and 1 have the same multiplicities. 
Proof. Let $z \in \mathbb{C} \backslash \sum_{K}$ be an eigenvalue of the operator $H(K)$ and $f=\left(f_{0}, f_{1}, f_{2}\right) \in \mathcal{H}$ be the corresponding eigenvector. Then $f_{0}, f_{1}$ and $f_{2}$ satisfy the system of equations:

$$
\begin{aligned}
& \left(w_{0}(K)-z\right) f_{0}+\int_{\mathbb{T}^{\mathrm{d}}} v_{0}(t) f_{1}(t) d t=0 \\
& v_{0}(p) f_{0}+\left(w_{1}(K ; p)-z\right) f_{1}(p)+\int_{\mathbb{T}^{\mathrm{d}}} v_{1}(t) f_{2}(p, t) d t=0 ; \\
& \frac{1}{2}\left(v_{1}(p) f_{1}(q)+v_{1}(q) f_{1}(p)\right)+\left(w_{2}(K ; p, q)-z\right) f_{2}(p, q)=0 .
\end{aligned}
$$

The condition $z \notin\left[m_{K}, M_{K}\right]$ yields that the inequality $w_{2}(K ; p, q)-z \neq 0$ holds for all $p, q \in \mathbb{T}^{\mathrm{d}}$. Then, from the third equation of the system (5.1) for $f_{2}$ we have:

$$
f_{2}(p, q)=-\frac{v_{1}(q) f_{1}(p)+v_{1}(p) f_{1}(q)}{2\left(w_{2}(K ; p, q)-z\right)} .
$$

Substituting the expression (5.2) for $f_{2}$ into the second equation of the system (5.1), we obtain that the system of equations:

$$
\begin{aligned}
& \left(w_{0}(K)-z\right) f_{0}+\int_{\mathbb{T}^{\mathrm{d}}} v_{0}(t) f_{1}(t) d t=0 ; \\
& v_{0}(p) f_{0}+\Delta_{K}(p ; z) f_{1}(p)-\frac{v_{1}(p)}{2} \int_{\mathbb{T}^{\mathrm{d}}} \frac{v_{1}(t) f_{1}(t) d t}{w_{2}(K ; p, t)-z}=0
\end{aligned}
$$

has a nontrivial solution and this system of equations has a nontrivial solution if and only if the system of equations (5.1) has a nontrivial solution.

By the definition of the set $\Lambda_{K}$ the inequality $\Delta_{K}(p ; z) \neq 0$ holds for all $z \notin \Lambda_{K}$ and $p \in \mathbb{T}^{\mathrm{d}}$. Therefore, the system of equations (5.3) has a nontrivial solution if and only if the following system of equations:

$$
\begin{aligned}
& f_{0}=\left(1+w_{0}(K)-z\right) f_{0}+\int_{\mathbb{T}^{d}} v_{0}(t) f_{1}(t) d t \\
& f_{1}(p)=-\frac{v_{0}(p) f_{0}}{\Delta_{K}(p ; z)}+\frac{v_{1}(p)}{2 \Delta_{K}(p ; z)} \int_{\mathbb{T}^{d}} \frac{v_{1}(t) f_{1}(t) d t}{w_{2}(K ; p, t)-z}
\end{aligned}
$$

or $2 \times 2$ matrix equation:

$$
g=T(z) g, \quad g=\left(f_{0}, f_{1}\right) \in \mathcal{H}_{0} \oplus \mathcal{H}_{1}
$$

has a nontrivial solution and the linear subspaces of solutions of (5.1) and (5.4) have the same dimension.

Remark 5.2. We point out that the matrix equation 5.4 is an analog of the Faddeev type system of integral equations for eigenfunctions of the operator $H(K)$ and it is played crucial role in the analysis of the spectrum of $H(K)$.

\section{Essential spectrum and its new branches}

In this section, applying the statements of Sections 3-5, the Weyl criterion [21] we investigate the essential spectrum of $H(K)$.

We denote by $\|\cdot\|$ and $(\cdot, \cdot)$ the norm and scalar product in the corresponding Hilbert spaces.

For the convenience of the reader we formulate Weyl's criterion for the essential spectrum of $H(K)$ as follows. First, a number $\lambda$ is in the spectrum of $H(K)$ if and only if there exists a sequence $\left\{F_{n}(K)\right\}$ in the space $\mathcal{H}$ such that $\left\|F_{n}(K)\right\|=1$ and:

$$
\lim _{n \rightarrow \infty}\left\|\left(H(K)-z_{0}(K) E\right) F_{n}(K)\right\|=0
$$

Here, $E$ is an identity operator on $\mathcal{H}$. Furthermore, $\lambda$ is in the essential spectrum if there is a sequence satisfying this condition, but such that it contains no convergent subsequence (this is the case if, for example $\left\{F_{n}(K)\right\}$ is an orthonormal sequence); such a sequence is called a singular sequence.

The following theorem describes the location of the essential spectrum of $H(K)$.

Theorem 6.1. The essential spectrum of $H(K)$ is coincide with the spectrum of $H_{\mathrm{ch}}(K)$, that is, $\sigma_{\mathrm{ess}}(H(K))=$ $\sigma\left(H_{\mathrm{ch}}(K)\right)$. Moreover the set $\sigma_{\mathrm{ess}}(H(K))$ consists no more than three bounded closed intervals. 
Proof. By the Theorem 4.1, we have $\sigma\left(H_{\mathrm{ch}}(K)\right)=\Sigma_{K}$. Therefore, we must to show that $\sigma_{\text {ess }}(H(K))=\Sigma_{K}$. We begin by proving $\Sigma_{K} \subset \sigma_{\text {ess }}(H(K))$. Since, the set $\Sigma_{K}$ has form $\Sigma_{K}=\Lambda_{K} \cup\left[m_{K} ; M_{K}\right]$ first we show that $\left[m_{K} ; M_{K}\right] \subset \sigma_{\text {ess }}(H(K))$. Let $z_{0}(K) \in\left[m_{K} ; M_{K}\right]$ be an arbitrary point. We prove that $z_{0}(K) \in \sigma_{\text {ess }}(H(K))$. To this end it is suffices to construct a sequence of orthonormal vector-functions $\left\{F_{n}(K)\right\} \subset \mathcal{H}$ satisfying (6.1).

From continuity of the function $w_{2}(K ; \cdot, \cdot)$ on the compact set $\left(\mathbb{T}^{\mathrm{d}}\right)^{2}$ it follows that there exists some point $\left(p_{0}(K), q_{0}(K)\right) \in\left(\mathbb{T}^{\mathrm{d}}\right)^{2}$ such that $z_{0}(K)=w_{2}\left(K ; p_{0}(K), q_{0}(K)\right)$.

For $n \in \mathbb{N}$ we consider the following vicinity of the point $\left(p_{0}(K), q_{0}(K)\right) \in\left(\mathbb{T}^{\mathrm{d}}\right)^{2}$ :

$$
W_{n}(K):=V_{n}\left(p_{0}(K)\right) \times V_{n}\left(q_{0}(K)\right),
$$

where:

$$
V_{n}\left(p_{0}(K)\right):=\left\{p \in \mathbb{T}^{\mathrm{d}}: \frac{1}{n+n^{\prime}+1}<\left|p-p_{0}(K)\right|<\frac{1}{n+n^{\prime}}\right\}
$$

is the punctured neighborhood of the point $p_{0}(K) \in \mathbb{T}^{\mathrm{d}}$ and $n^{\prime} \in \mathbb{N}$ is chosen in such way that $V_{n}\left(p_{0}(K)\right) \cap$ $V_{n}\left(q_{0}(K)\right)=\emptyset$ for all $n \in \mathbb{N}$ (provided that $p_{0}(K) \neq q_{0}(K)$ ).

Let $\mu(\Omega)$ be the Lebesgue measure of the set $\Omega$ and $\chi_{\Omega}(\cdot)$ be the characteristic function of the set $\Omega$. We choose the sequence of functions $\left\{F_{n}(K)\right\} \subset \mathcal{H}$ as follows:

$$
F_{n}(K):=\frac{1}{\sqrt{2 \mu\left(W_{n}(K)\right)}}\left(\begin{array}{c}
0 \\
0 \\
\chi_{W_{n}(K)}(p, q)+\chi_{W_{n}(K)}(q, p)
\end{array}\right) .
$$

It is clear that $\left\{F_{n}(K)\right\}$ is an orthonormal sequence.

For any $n \in \mathbb{N}$ let us consider an element $\left(H(K)-z_{0}(K) E\right) F_{n}(K)$ and estimate its norm:

$$
\left\|\left(H(K)-z_{0}(K) E\right) F_{n}(K)\right\|^{2} \leq \sup _{(p, q) \in W_{n}(K)}\left|w_{2}(K ; p, q)-z_{0}(K)\right|^{2}+\mu\left(V_{n}\left(p_{0}(K)\right)\right) \max _{p \in \mathbb{T}^{\mathrm{d}}}\left|v_{1}(p)\right|^{2} .
$$

The construction of the set $V_{n}\left(p_{0}(K)\right)$ and the continuity of the function $w_{2}(K ; \cdot, \cdot)$ implies $\left\|\left(H(K)-z_{0}(K) E\right) F_{n}(K)\right\| \rightarrow 0$ as $n \rightarrow \infty$, i.e. $z_{0}(K) \in \sigma_{\text {ess }}(H(K))$. Since the point $z_{0}(K)$ is an arbitrary we have $\left[m_{K} ; M_{K}\right] \subset \sigma_{\text {ess }}(H(K))$.

Now, let us prove that $\Lambda_{K} \subset \sigma_{\text {ess }}(H(K))$. Taking an arbitrary point $z_{1}(K) \in \Lambda_{K}$ we show that $z_{1}(K) \in$ $\sigma_{\text {ess }}(H(K))$. Two cases are possible: $z_{1}(K) \in\left[m_{K} ; M_{K}\right]$ or $z_{1}(K) \notin\left[m_{K} ; M_{K}\right]$. If $z_{1}(K) \in\left[m_{K} ; M_{K}\right]$, then it is already proven above that $z_{1}(K) \in \sigma_{\text {ess }}(H(K))$. Let $z_{1}(K) \in \Lambda_{K} \backslash\left[m_{K} ; M_{K}\right]$. Definition of the set $\Lambda_{K}$ and Lemma 3.2 imply that there exists a point $p_{1}(K) \in \mathbb{T}^{\mathrm{d}}$ such that $\Delta_{K}\left(p_{1}(K) ; z_{1}(K)\right)=0$.

We choose a sequence of orthogonal vector-functions $\left\{\Phi_{n}(K)\right\}$ as

$$
\begin{aligned}
\Phi_{n}(K):=\left(\begin{array}{c}
0 \\
\phi_{1}^{(n)}(K ; p) \\
\phi_{2}^{(n)}(K ; p, q)
\end{array}\right), \quad \text { where } \quad \phi_{1}^{(n)}(K ; p) & :=\frac{c_{n}(K ; p) \chi_{V_{n}\left(p_{1}(K)\right)}(p)}{\sqrt{\mu\left(V_{n}\left(p_{1}(K)\right)\right)}}, \\
\phi_{2}^{(n)}(K ; p, q) & :=-\frac{v_{1}(p) \phi_{1}^{(n)}(K ; q)+v_{1}(q) \phi_{1}^{(n)}(K ; p)}{2\left(w_{2}(K ; p, q)-z_{1}(K)\right)} .
\end{aligned}
$$

Here, $c_{n}(K ; p) \in L_{2}\left(\mathbb{T}^{\mathrm{d}}\right)$ is chosen from the orthonormality condition for $\left\{\Phi_{n}(K)\right\}$, that is, from the condition:

$$
\begin{aligned}
\left(\Phi_{n}(K), \Phi_{m}(K)\right) & =\frac{1}{2 \sqrt{\mu\left(V_{n}\left(p_{1}(K)\right)\right)} \sqrt{\mu\left(V_{m}\left(p_{1}(K)\right)\right)}} \\
& \times \int_{V_{n}\left(p_{1}(K)\right) V_{m}\left(p_{1}(K)\right)} \frac{v_{1}(p) v_{1}(q) c_{n}(K ; p) c_{m}(K ; q)}{\left(w_{2}(K ; p, q)-z_{1}(K)\right)^{2}} d p d q=0
\end{aligned}
$$

for $n \neq m$. The existence of $\left\{c_{n}(K ; \cdot)\right\}$ is a consequence of the following proposition.

Proposition 6.2. There exists an orthonormal system $\left\{c_{n}(K ; \cdot)\right\} \subset L_{2}\left(\mathbb{T}^{\mathrm{d}}\right)$ satisfying the conditions $\operatorname{supp} c_{n}(K ; \cdot) \subset$ $V_{n}\left(p_{1}(K)\right)$ and $(6.2)$.

Proof of Proposition 6.2. We construct the sequence $\left\{c_{n}(K ; \cdot)\right\}$ by the induction method. Suppose that $c_{1}(K ; p):=$ $\chi_{V_{1}\left(p_{1}(K)\right)}(p)\left(\sqrt{\mu\left(V_{1}\left(p_{1}(K)\right)\right)}\right)^{-1}$. Now, we choose the function $\widetilde{c}_{2}(K ; \cdot) \in L_{2}\left(V_{2}\left(p_{1}(K)\right)\right)$ so that $\left\|\widetilde{c}_{2}(K ; \cdot)\right\|=$ 1 and $\left(\widetilde{c}_{2}(K ; \cdot), \varepsilon_{1}^{(2)}(K ; \cdot)\right)=0$, where

$$
\varepsilon_{1}^{(2)}(K ; p):=v_{1}(p) \chi_{V_{2}\left(p_{1}(K)\right)}(p) \int_{\mathbb{T}^{\mathrm{d}}} \frac{v_{1}(q) c_{1}(K ; q) d q}{\left(w_{2}(K ; p, q)-z_{1}(K)\right)^{2}} .
$$


We set $c_{2}(K ; p):=\widetilde{c}_{2}(K ; p) \chi_{V_{1}\left(p_{1}(K)\right)}(p)$. We continue this process. Suppose that $c_{1}(K ; p), \ldots, c_{n}(K ; p)$ are constructed. Then the function $\widetilde{c}_{n+1}(K ; \cdot) \in L_{2}\left(V_{n+1}\left(p_{1}(K)\right)\right)$ is chosen so that it is orthogonal to all functions:

$$
\varepsilon_{m}^{(n+1)}(K ; p):=v_{1}(p) \chi_{V_{n+1}\left(p_{1}(K)\right)}(p) \int_{\mathbb{T}^{\mathrm{d}}} \frac{v_{1}(q) c_{m}(K ; q) d q}{\left(w_{2}(K ; p, q)-z_{1}(K)\right)^{2}}, \quad m=1, \ldots, n
$$

and $\left\|\widetilde{c}_{n+1}(K ; \cdot)\right\|=1$. Let $c_{n+1}(K ; p):=\widetilde{c}_{n+1}(K ; p) \chi_{V_{n+1}\left(p_{1}(K)\right)}(p)$. Thus, we have constructed the orthonormal system of functions $\left\{c_{n}(K ; \cdot)\right\}$ satisfying the assumptions of the proposition. Proposition 6.2 is proved.

We continue the proof of Theorem 6.1. Now for $n \in \mathbb{N}$ we consider $\left(H(K)-z_{1}(K) E\right) \Phi_{n}(K)$ and estimate its norm as:

$$
\begin{aligned}
\left\|\left(H(K)-z_{1}(K) E\right) \Phi_{n}(K)\right\|^{2} & \leq C(K) \max _{p \in \mathbb{T}^{d}} v_{1}^{2}(p) \mu\left(V_{n}\left(p_{1}(K)\right)\right) \\
& +2 \sup _{p \in V_{n}\left(p_{1}(K)\right)}\left|\Delta_{K}\left(p ; z_{1}(K)\right)\right|^{2}
\end{aligned}
$$

for some $C(K)>0$.

Since $\mu\left(V_{n}\left(p_{1}(K)\right)\right) \rightarrow 0$ and $\sup _{p \in V_{n}\left(p_{1}(K)\right)}\left|\Delta_{K}\left(p ; z_{1}(K)\right)\right|^{2} \rightarrow 0$ as $n \rightarrow \infty$, from the (6.3) it follows that $\left\|\left(H(K)-z_{1}(K) E\right) \Phi_{n}(K)\right\| \rightarrow 0$ as $n \rightarrow \infty$. This implies $z_{1}(K) \in \sigma_{\text {ess }}(H(K))$. Since the point $z_{1}(K)$ is an arbitrary, we have $\Lambda_{K} \subset \sigma_{\text {ess }}(H(K))$. Therefore, we proved that $\Sigma_{K} \subset \sigma_{\text {ess }}(H)$.

Now, we prove the inverse inclusion, i.e. $\sigma_{\text {ess }}(H(K)) \subset \Sigma_{K}$. Since for each $z \in \mathbb{C} \backslash \Sigma_{K}$ the operator $T(K ; z)$ is a compact-operator-valued function on $\mathbb{C} \backslash \Sigma_{K}$, from the self-adjointness of $H(K)$ and Theorem 5.1 it follows that the operator $(I-T(K, z))^{-1}$ exists if $z$ is real and has a large absolute value. The analytic Fredholm theorem (see, e.g., Theorem VI.14 in [21]) implies that there is a discrete set $S_{K} \subset \mathbb{C} \backslash \Sigma_{K}$ such that the function $(I-T(K, z))^{-1}$ exists and is analytic on $\mathbb{C} \backslash\left(S_{K} \cup \Sigma_{K}\right)$ and is meromorphic on $\mathbb{C} \backslash \Sigma_{K}$ with finite-rank residues. This implies that the set $\sigma(H(K)) \backslash \Sigma_{K}$ consists of isolated points, and the only possible accumulation points of $\Sigma_{K}$ can be on the boundary. Thus:

$$
\sigma(H(K)) \backslash \Sigma_{K} \subset \sigma_{\text {disc }}(H(K))=\sigma(H(K)) \backslash \sigma_{\text {ess }}(H(K)) .
$$

Therefore, the inclusion $\sigma_{\text {ess }}(H(K)) \subset \Sigma_{K}$ holds. Finally we obtain the equality $\sigma_{\text {ess }}(H(K))=\Sigma_{K}$.

By Lemma 3.3 for any $K, k \in \mathbb{T}^{\text {d }}$ the operator $h(K, k)$ has no more than one simple eigenvalue on the 1.h.s. (resp. r.h.s) of $E_{\min }(K, k)$ (resp. $E_{\max }(K, k)$ ). Then, by the theorem on the spectrum of decomposable operators [21] and by the definition of the set $\Lambda_{K}$ it follows that the set $\Lambda_{K}$ consists of the union of no more than two bounded closed intervals. Therefore, the set $\Sigma_{K}$ consists of the union of no more than three bounded closed intervals. Theorem 6.1 is completely proved.

In the following we introduce the new subsets of the essential spectrum of $H(K)$.

Definition 6.3. The sets $\Lambda_{K}$ and $\left[m_{K} ; M_{K}\right]$ are called two- and three-particle branches of the essential spectrum of $H(K)$, respectively.

It is obvious that for a given $H(K)$, the operator $H_{\mathrm{ch}}(K)$ is uniquely selected by the property of decomposability into a direct integral.

According to Theorem 6.1, the operator $H_{\mathrm{ch}}(K)$ have the characteristic property of a channel operator of the corresponding discrete Schrödinger operator, see $[18,19]$. Therefore, we call this operator the channel operator associated with $H(K)$. We note that the channel operator $H_{\mathrm{ch}}(K)$ have a more simple structure than the operator $H(K)$, and hence, Theorem 6.1 plays an important role in the subsequent investigations of the essential spectrum of $H(K)$.

Since for any $K \in \mathbb{T}^{\mathrm{d}}$ and $z \in \mathbb{C} \backslash \Sigma_{K}$ the operators $T_{00}(K, z), T_{01}(K, z)$ and $T_{10}(K, z)$ are one dimensional and the kernel of $T_{11}(K, z)$ is a continuous function on $\left(\mathbb{T}^{\mathrm{d}}\right)^{2}$, the Fredholm determinant $\Omega_{K}(z)$ of the operator $I-T(K, z)$, where $I$ is the identity operator in $\mathcal{H}_{0} \oplus \mathcal{H}_{1}$, exists and is a real-analytic function on $\mathbb{C} \backslash \Sigma_{K}$.

According to Fredholm's theorem [21] and Theorem 5.1 the number $z \in \mathbb{C} \backslash \Sigma_{K}$ is an eigenvalue of $H(K)$ if and only if $\Omega_{K}(z)=0$, that is:

$$
\sigma_{\text {disc }}(H(K))=\left\{z \in \mathbb{C} \backslash \Sigma_{K}: \Omega_{K}(z)=0\right\} .
$$

\section{References}

[1] Minlos R.A., Spohn H. The three-body problem in radioactive decay: the case of one atom and at most two photons. Topics in Statistical and Theoretical Physics. Amer. Math. Soc. Transl., Ser. 2, 177, AMS, Providence, RI, 1996, P. 159-193.

[2] Mogilner A.I. Hamiltonians in solid state physics as multiparticle discrete Schrödinger operators: problems and results. Advances in Sov. Math., 1991, 5, P. 139-194.

[3] Friedrichs K.O. Perturbation of spectra in Hilbert space. Amer. Math. Soc. Providence, Rhole Island, 1965. 
[4] Hübner M., Spohn H. Spectral properties of the spin-boson Hamiltonian. Ann. Inst. H. Poincaré. Phys. Théor., 1995, 62(3), P. 289-323.

[5] Hübner M., Spohn H. Atom interacting with photons: an N-body Schrödinger problem. Tech. rep., 1994.

[6] Muminov M., Neidhardt H., Rasulov T. On the spectrum of the lattice spin-boson Hamiltonian for any coupling: 1D case. Journal of Mathematical Physics, 2015, 56, P. 053507.

[7] Rasulov T.Kh. Branches of the essential spectrum of the lattice spin-boson model with at most two photons. Theoretical and Mathematical Physics, 2016, 186(2), P. 251-267.

[8] Zhukov Yu.V., Minlos R.A. The spectrum and scattering in the "spin-boson" model with at most three photons. Theor. Math. Phys., 1995, 103(1), P. 63-81.

[9] Sigal I.M., Soffer A., Zielinski L. On the spectral properties of Hamiltonians without conservation of the particle number. J. Math. Phys., 2002, 42(4), P. 1844-1855.

[10] Lakaev S.N., Rasulov T.Kh. A model in the theory of perturbations of the essential spectrum of multi-particle operators. Math. Notes, 2003, 73(4), P. 521-528.

[11] Muminov M.I., Rasulov T.Kh. An eigenvalue multiplicity formula for the Schur complement of a $3 \times 3$ block operator matrix. Siberian Math. $J ., 2015, \mathbf{5 6}(4)$, P. 878-895.

[12] Rasulov T.H. Investigations of the essential spectrum of a Hamiltonian in Fock space. Appl. Math. Inf. Sci., 2010, 4(3), P. 395-412.

[13] Rasulov T.H., Muminov M.I., Hasanov M. On the spectrum of a model operator in Fock space. Methods Funct. Anal. Topology, 2009, 15(4), P. 369-383.

[14] Albeverio S., Lakaev S.N., Rasulov T.H. On the spectrum of an Hamiltonian in Fock space. Discrete spectrum asymptotics. J. Stat. Phys., 2007, 127(2), P. 191-220.

[15] Albeverio S., Lakaev S.N., Rasulov T.H. The Efimov effect for a model operator associated with the Hamiltonian of a non conserved number of particles. Methods Funct. Anal. Topology, 2007, 13(1), P. 1-16.

[16] Muminov M.I., Rasulov T.H. On the number of eigenvalues of the family of operator matrices. Nanosystems: Physics, Chemistry, Mathematics, 2014, 5(5), P. 619-626.

[17] Feynman R.P. Statistical Mechanics: A Set of Lectures (2nd ed.). Reading, Massachusetts: Addison-Wesley, 1998.

[18] Lakaev S.N., Muminov M.É. Essential and discrete spectra of the three-particle Schrödinger operator on a lattices. Theor. Math. Phys., 2003, 135(3), P. 849-871.

[19] Albeverio S., Lakaev S.N., Muminov Z.I. On the structure of the essential spectrum for the three-particle Schrödinger operators on lattices. Math. Nach., 2007, 280, P. 699-716.

[20] Rasulov T.H., Dilmurodov E.B. Eigenvalues and virtual levels of a family of $2 \times 2$ operator matrices. Methods Funct. Anal. Topology, 2019, 25(3), P. 273-281.

[21] Reed M., Simon B. Methods of modern mathematical physics. IV: Analysis of Operators. Academic Press, New York, 1979.

[22] Malishev V.A., Minlos R.A. Linear infinite-particle operators. Translations of Mathematical Monographs. 143, AMS, Providence, RI, 1995. 